Anestesiología

Abril-Junio 2022

Vol. 45. No. 2. pp 92-100

doi: $10.35366 / 103883$

\section{Lámina de plástico vs caja de acrílico como dispositivo de barrera durante intubación endotraqueal}

\author{
Plastic sheet vs. acrylic box as barrier device during endotracheal intubation \\ Dr. Ernesto Jose Cortes-Ospino,* Dra. Mirna Magali Delgado-Carlo, ${ }^{\ddagger}$ \\ Dr. Miguel Pineda-Sánchez ${ }^{\S}$
}

Citar como: Cortes-Ospino EJ, Delgado-Carlo MM, Pineda-Sánchez M. Lámina de plástico vs caja de acrílico como dispositivo de barrera durante intubación endotraqueal. Rev Mex Anestesiol. 2022; 45 (2): 92-100. https://dx.doi. org/10.35366/103883

RESUMEN. Introducción: La utilización de dispositivos de barrera adicional para la intubación endotraqueal constituye un reto para la práctica médica durante la actual pandemia. Se pueden utilizar múltiples herramientas para intubación del paciente minimizando la exposición del operador sin afectar la seguridad del procedimiento. El médico debe evaluar y comprender adecuadamente cualquier estrategia adicional, ya que podría interferir con la intubación, aumentando el tiempo de manipulación. Objetivo: Comparar preferencia de los anestesiólogos entre dos dispositivos de barrera, uno diseñado con base en una lámina plástica versus caja de acrílico. Material y métodos: Incluimos 102 pacientes intervenidos de cirugías electivas y de urgencias que aceptaron participar en este estudio prospectivo, transversal y comparativo de manera voluntaria con previo consentimiento informado. Resultados: La lámina de plástico permite un menor tiempo de intubación, menos intentos de laringoscopía y disminuye dificultades de abordaje, además genera un grado de satisfacción mayor en el personal en comparación con dispositivos rígidos como la caja de acrílico. Conclusiones: Es posible obtener buenos resultados para instrumentar la vía aérea cuando se utilizan dispositivos de barrera adicional en personal entrenado. No obstante, teniendo en cuenta los hallazgos de este trabajo existen ventajas con el uso de lámina de plástico.

ABSTRACT. Introduction: The use of additional barrier devices for endotracheal intubation constitutes a challenge for medical practice during the current pandemic. Multiple tools can be used for patient intubation minimizing operator exposure without compromising procedural safety. Any additional strategy must be properly evaluated and understood by the physician as it could interfere with intubation, increasing handling time. Objective: To compare anesthesiologists' preference between two barrier devices, one designed based on a plastic sheet vs an acrylic box. Material and methods: We included 102 patients who underwent elective and emergency surgeries who voluntarily agreed to participate in this prospective, cross-sectional and comparative study with prior informed consent. Results: The plastic sheet allows a shorter intubation time, fewer laryngoscopy attempts and reduces approach difficulties, in addition it generates a higher degree of satisfaction in the personnel compared to rigid devices such as the acrylic box. Conclusions: It is possible to obtain good results to instrument the airway when additional barrier devices are used in trained personnel. However, taking into account the findings of this work, there are advantages with the use of plastic sheeting.

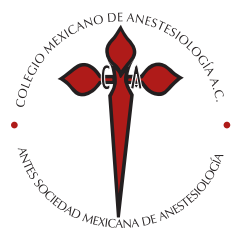

Palabras clave: Anestesia, COVID-19, intubación endotraqueal, caja de acrílico, lámina plástica, barrera adicional de protección.

Keywords: Anesthesia, COVID-19, endotracheal intubation, acrylic box, plastic sheet, additional barrier of protection.

* Médico Epidemiólogo y Residente de tercer año de Anestesiología.

‡ Médico Anestesiólogo y

Algóloga Paliativista.

$\S$ Médico Anestesiólogo Jefe del Servicio de Anestesiología.

Hospital Regional «General Ignacio Zaragoza» Instituto de Seguridad y Servicios Sociales de los Trabajadores del Estado ISSSTE. Ciudad de México, México.

Correspondencia:

Dr. Ernesto Jose Cortes-Ospino

Calzada Ignacio Zaragoza

1711, Colonia

Ejército constitucionalista,

CP 09220, Iztapalapa,

Ciudad de México.

E-mail:md.ernestocortes@

gmail.com

Recibido: $17-08-2020$

Aceptado: 05-10-2021

\section{INTRODUCCIÓN}

C on una inminente escasez de equipos de protección personal (EPP) y un aumento en el número de contagios entre el personal de atención médica, la transmisión del coronavirus a través de la aerosolización está promoviendo nuevas implementaciones en el equipo de barrera para la protección del trabajador de salud al realizar la intubación orotraqueal $^{(1)}$.
A fines de 2019, se detectó por primera vez una neumonía de causa desconocida, después se identificó que fue causada por este agente en China. El Comité Internacional de Taxonomía de los virus lo denominó «coronavirus 2 del síndrome respiratorio agudo severo», o SARS-CoV-2, ya que está relacionado con el virus que causó el brote del «Síndrome respiratorio agudo severo» (SARS por sus siglas en inglés) en $2003^{(2)}$. El 11 de febrero de 2020, la Organización Mundial de la Salud (OMS) anunció que el nombre oficial de la nueva 
enfermedad que originó sería «COVID-19», una versión abreviada de enfermedad por coronavirus $2019^{(3)}$.

El manejo de la vía aérea de los pacientes sospechosos o confirmados con COVID-19 presenta un riesgo significativo para el personal involucrado debido a la generación y presencia de aerosoles en la intervención de la vía aérea ${ }^{(4)}$. La aerosolización puede ocurrir durante la colocación de una mascarilla facial, en la ventilación de la vía aérea, durante la intubación, extubación y en la reanimación cardiopulmonar ${ }^{(5)}$. Estas actividades requieren mayores precauciones y contención de partículas virales respiratorias. Se ha demostrado que un sistema de contención puede limitar el rociado de una fracción significativa de partículas respiratorias durante una tos o extubación simulada ${ }^{(6,7)}$.

Se hace necesario entonces el uso de herramientas para reducir la transmisión de aerosoles y micro gotas de los pacientes con enfermedad por coronavirus 2019 (COVID-19). Brown y colegas ${ }^{(8)}$ y Babazade y colaboradores ${ }^{(1)}$ describen el uso de cortinas de plástico, mientras que Lai y Chang ${ }^{(9)}$ y Tsai ${ }^{(10)}$ describen diseños de cajas rígidas. Aunque estas propuestas son innovadoras su utilización puede generar algunos inconvenientes significativos ${ }^{(11)}$.

Se están utilizado recientemente cajas de acrílico en la mayoría de las instituciones en nuestro país, sin embargo, existe suficiente evidencia en la literatura a nivel mundial que pone en manifiesto la restricción del movimiento de las manos y la necesidad de entrenamiento adicional, se plantea como alternativa un método de barrera hecho de una lámina de plástico transparente de fácil acceso, barato y reusable. No hay estudios comparativos entre ambas herramientas de barrera en nuestra población.

\section{Antecedentes}

La nueva enfermedad por coronavirus humano COVID-19 se ha convertido en la quinta pandemia documentada en la historia $^{(12)}$. El 31 de diciembre de 2019, el gobierno chino informó por primera vez un brote de la enfermedad por coronavirus en Wuhan, la capital de la provincia de Hubei en China ${ }^{(13)}$. El 11 de marzo de 2020, la OMS finalmente alertó al mundo de COVID-19 como una pandemia. Después de la gripe española de 1918 (H1N1), la gripe asiática de 1957 (H2N2), la gripe de Hong Kong de 1968 (H3N2) y la gripe pandémica de 2009 (H1N1), que causó aproximadamente 50 millones, 1.5 millones, 1 millón y 300,000 muertes humanas, respectivamente ${ }^{(14-17)}$.

Todos los casos de infección y epidemias recientes muestran que los coronavirus imponen una amenaza continua para los seres humanos y la economía en relación a su forma inesperada de aparición, se propagan fácilmente y tienen consecuencias catastróficas ${ }^{(18)}$. La pandemia actual por coronavirus ha desafiado la atención médica a nivel mundial, dando como resultado al 20 de mayo de 2020, a nivel mundial se tenían 4'789,205 casos confirmados de SARS-CoV-2 y 318,789 defunciones con una tasa de letalidad global de $6.7 \%$. La actualización al 31 de Julio de 2020 informa de 17.106.007 casos confirmados a nivel mundial y 668,910 muertes $^{(19)}$.

Su transmisibilidad es muy alta (al nivel de SARS o de la pandemia gripal de 1918) sugiriendo un posible crecimiento exponencial ${ }^{(20)}$. El mecanismo es por contacto próximo, gotas respiratorias o manos contaminadas, también es posible a través de superficies infectadas ${ }^{(21)}$. Los coronavirus humanos persisten en metales, vidrio o plásticos más de nueve días. No se ha descartado un mecanismo fecal-oral. Es determinante la transmisión nosocomial, especialmente para los trabajadores del área de la salud ${ }^{(21)}$.

La propagación de la enfermedad aumenta en un período asintomático relativamente largo que varía de ocho a 15 días, pero puede ser de hasta 24 días $^{(22)}$. El número esperado de casos secundarios derivados de un individuo infectado es de aproximadamente dos a tres personas como mínimo ${ }^{(20)}$. Se considera que muchos pacientes pueden estar asintomáticos por lo que cobra importancia tomar medidas de precaución al realizar procedimientos generadores de aerosoles, como lo es la intubación orotraqueal en todos los pacientes.

Es muy probable que pacientes con confirmación o sospecha de COVID-19 precisen de una intervención quirúrgica. Los escenarios en que se contemplan son intervenciones urgentes y electivas no demorables ${ }^{(23)}$. La indicación de cirugía urgente se debe individualizar en todos los casos y tratar de realizarse basándose en un diagnóstico con la mayor certeza posible ${ }^{(23)}$. Los trabajadores de la salud son una ruta principal de transmisión, similar a los virus relacionados que causan SARS y MERS ${ }^{(24)}$. Los anestesiólogos tienen un mayor riesgo de exposición durante el tratamiento perioperatorio a través del contacto directo, especialmente la intubación traqueal. La transmisión puede ocurrir desde individuos infectados asintomáticos. Por lo tanto, el personal de la salud debe considerar a todos los pacientes como una posible fuente de infección a menos que se demuestre lo contrario(25).

Esto es particularmente cierto para los anestesiólogos que comúnmente están en contacto cercano con pacientes y entran en contacto con aerosoles y secreciones de las vías respiratorias; en particular, se encuentra con este virus en la sala de operaciones (SO), la Unidad de Cuidados Intensivos (UCI) y durante las intubaciones urgentes o de emergencia. Los pacientes se presentan principalmente con manifestaciones pulmonares, que con frecuencia requieren soporte avanzado de oxígeno, incluida una vía aérea avanzada ${ }^{(26)}$.

Debido a la naturaleza altamente contagiosa de COVID-19, la mayor preocupación del personal médico se ha centrado en minimizar la exposición durante la intubación. Las medidas profilácticas han incluido la colocación de equipo de protección personal (EPP) adecuado y el uso de videolaringoscopios 
para mantener la distancia de la vía aérea ${ }^{(27)}$. Así como también para reducir la potencial dificultad del acceso a la misma. Aunque la reducción de la exposición del personal médico es de evidente importancia, la literatura que discute estrategias clínicas durante la inducción e intubación de pacientes con COVID-19 es escasa. Es importante destacar que optimizar el proceso de intubación podría reducir la exposición al disminuir el tiempo del contacto con la vía aérea del paciente ${ }^{(27)}$.

Se pueden implementar numerosas estrategias farmacológicas durante la inducción e intubación de COVID-19. Cualquier estrategia debe incorporar una inducción de secuencia rápida para minimizar la exposición y evitar la aspiración. Aunque ningún estudio ha evaluado o determinado los agentes ideales para inducir a estos pacientes, se puede inferir mucho de la fisiopatología de la enfermedad. Las categorías de agentes farmacológicos incluyen analgésicos, hipnóticos y bloqueadores neuromusculares ${ }^{(27)}$.

La intubación emergente de la vía aérea con frecuencia requiere bloqueo neuromuscular, es específicamente útil en pacientes con COVID-19 debido a la interrupción de la respiración y la tos, lo que disminuye la aerosolización del virus durante el procedimiento. Los agentes más utilizados en la práctica moderna incluyen rocuronio, vecuronio, cisatracurio y succinilcolina, siendo rocuronio de elección en inducción de secuencia rápida ${ }^{(27)}$.

\section{Dispositivos de barrera adicionales}

Cajas de acrílico: aunque estos diseños son innovadores, sufren algunos inconvenientes, la caja rígida limita significativamente los movimientos del antebrazo y la mano, por lo que se requiere de práctica para lograr eficiencia en su uso ${ }^{(9)}$. Además, el diseño rígido obliga al operador a situarse lejos del lado del paciente, lo que limita el manejo de la vía aérea ${ }^{(10)}$. también pueden tener el problema adicional que deben ser descontaminados, lo que podría ser un inconveniente en dispositivos que no son de grado médico ${ }^{(9)}$.

Láminas de plástico: diseños de cubiertas plásticas para cortinas de Brown y colegas ${ }^{(8)}$ y Babazade y colaboradores ${ }^{(1)}$ tienen la ventaja de ser económicos, comúnmente disponibles, rápidos de producir y desechables. También tienen múltiples puntos de acceso para asistencia; sin embargo, el peso de la cubierta plástica en las manos del operador puede ser un problema al realizar procedimientos bajo el dispositivo. La lámina de plástico se usó inicialmente bastante cerca de la cara del paciente y algunos pacientes no podían tolerarla ${ }^{(11)}$.

Para superar algunas de estas limitaciones, se han construido por ejemplo una capucha para encerrar la cabeza del paciente durante los procedimientos, este consiste en un soporte quirúrgico (mesa de mayo) sin la bandeja y la lámina de plástico de $1.60 \times 2.0 \mathrm{~m}$ de tamaño, formando una gran sábana de plástico transparente que permite una visualización clara de la cabeza del paciente. La lámina de plástico queda como una cortina, con una abertura dos aberturas de $10 \mathrm{~cm}$ frente a los antebrazos del operador, que permite su fácil movilización dentro del dispositivo. Esta herramienta mantiene las ventajas del diseño de barrera, ya que ofrece un espacio adecuado alrededor de la cabeza y el cuello del paciente, tanto para el laringoscopista como para el asistente de la vía aérea, permitiendo proporcionar un manejo efectivo de las vías respiratorias desde la preoxigenación hasta la extubación ${ }^{(11)}$.

El presente estudio clínico de tipo experimental prospectivo transversal comparativo, tiene por objetivo analizar la preferencia de los anestesiólogos al utilizar la lámina de plástico comparado con la caja de acrílico como dispositivo de barrera adicional para la intubación endotraqueal en pacientes intervenidos quirúrgicamente bajo anestesia general.

\section{MATERIAL Y MÉTODOS}

Tipo de estudio: clínico experimental prospectivo transversal comparativo.

Pacientes mayores de 18 años intervenidos quirúrgicamente bajo anestesia general; con instrumentación de la vía aérea en el Hospital Regional «General Ignacio Zaragoza», durante el período de estudio. Todos los participantes firmaron consentimiento informado.

Procedimos seleccionar a los pacientes en dos grupos (grupo 1 lámina de plástico versus grupo 2 caja de acrílico). El personal médico-quirúrgico fue provisto del equipo de protección personal adecuado, todos los pacientes ingresaron a sala con bata quirúrgica y cubreboca.

Previa monitorización del paciente, revisión de máquina de anestesia, verificación de dispositivos para la vía aérea y equipo de reanimación; se colocó la barrera de protección adicional correspondiente (lamina de plástico-caja de acrílico) cubriendo cabeza y cuello del paciente. Se utilizó anestesia general balanceada en ambos grupos de estudio, los pacientes recibieron para su inducción; secuencia rápida (un minuto) para su posterior laringoscopía.

A partir del primer minuto se inició la medición del tiempo de instrumentación por el personal auxiliar, este conteo finalizó hasta realizar el neumotaponamiento del globo de la cánula traqueal y habiendo corroborado capnometría en el monitor. A todos los pacientes se les realizó dicha laringoscopía con video (C-MAC) y los medicamentos y dosis empleadas para la inducción anestésica consistieron en fentanylo $3 \mu \mathrm{g} / \mathrm{kg} / \mathrm{do}-$ sis, rocuronio $1.2 \mathrm{mg} / \mathrm{kg} /$ dosis y propofol $1.5-2 \mathrm{mg} / \mathrm{kg} / \mathrm{dosis}$.

La observación posterior a la inducción anestésica consistió en una valoración de la escala de Cormack Lehane, duración de la laringoscopía, y número de intentos de la misma. Al finalizar el procedimiento se estableció una medición del grado de satisfacción, según el nivel de dificultad percibido por personal médico entrenado para la instrumentación de la vía aérea. En los casos de relajación muscular residual secun- 
daria (moderada, profunda o intensa) a las dosis empleadas de rocuronio, se administró sugammadex, en base al peso ideal, con dosis de 2, 4 y $16 \mathrm{mg} / \mathrm{kg}$ respectivamente, evaluando la respuesta clínica ${ }^{(28)}$. La fuente primaria de obtención datos de cada paciente fue interrogatorio directo, con la utilización del formato de captación de datos individual y su registro realizado en sala quirúrgica. La selección de las fuentes, métodos, técnicas y procedimientos en la recolección de la información se resume en el flujograma de decisión (Figura 1).

Definición del plan de procesamiento y presentación de la información: se realizó a través de Epi-info; estadística descriptiva con medidas de frecuencias y porcentajes para variables cualitativas y para variables cuantitativas medidas de resumen y dispersión (media y dispersión estándar) coe-

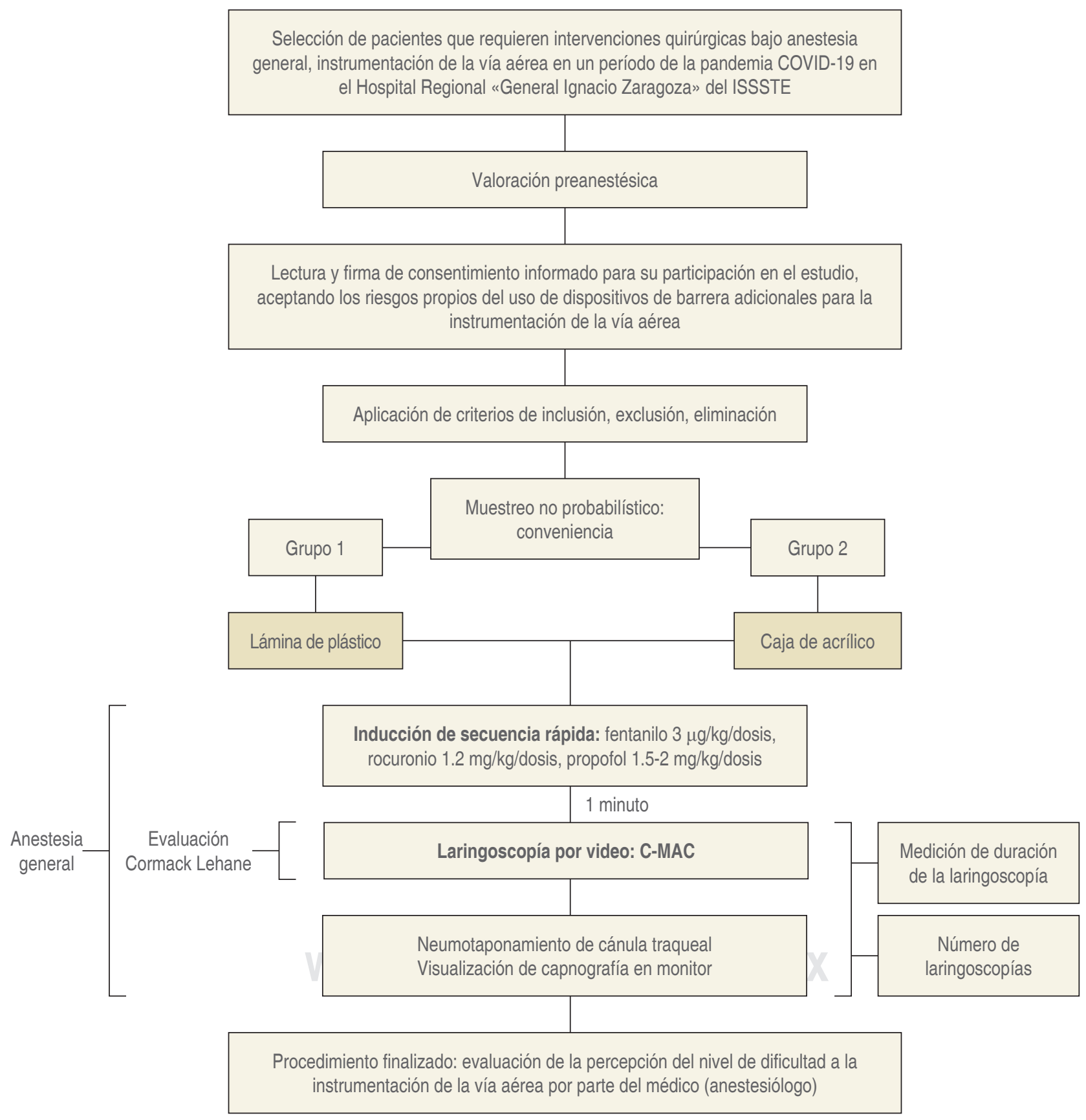

Figura 1: Flujograma de decisiones. 

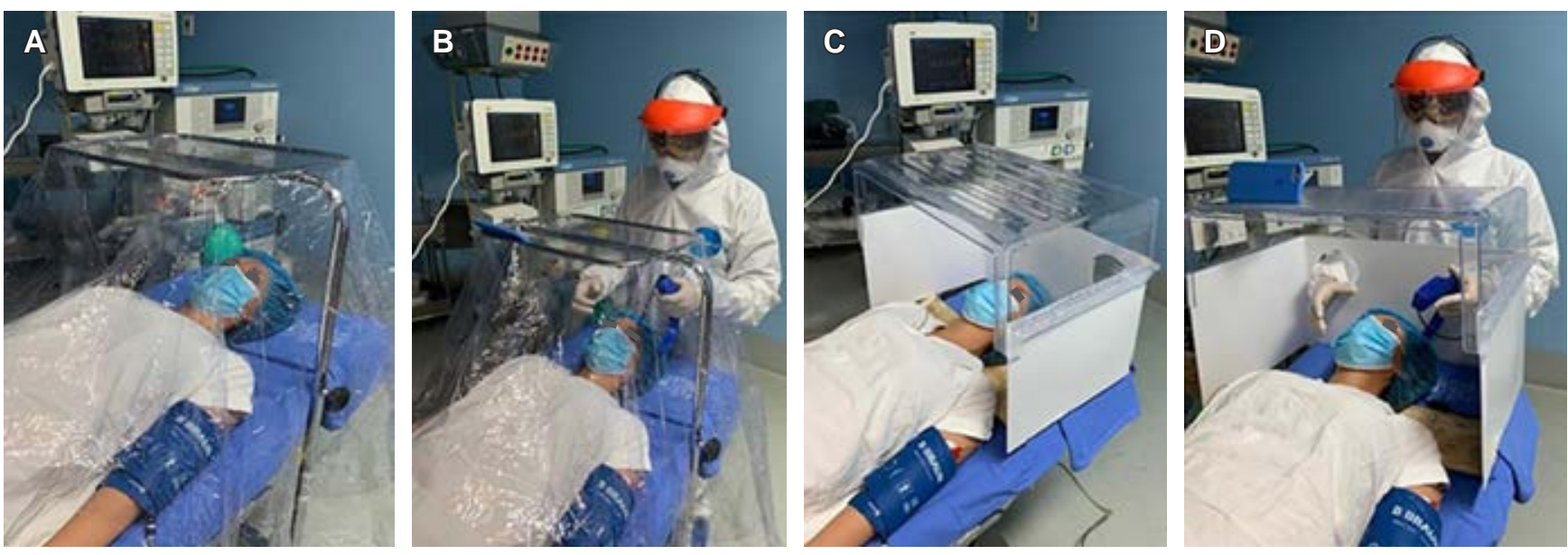

Figura 2: Dispositivos de barrera adicional. A y B) Dispositivo de barrera adicional tipo flexible para la intubación endotraqueal de lámina de plástico. C y D) Dispositivo de barrera rígido: caja de acrílico.

ficiente de correlación de Pearson. La diferencia de medias de las variables cualitativas entre grupos se analizó mediante $\chi^{z}$. El análisis se realizó a partir de las hojas de recolección de datos.

\section{RESULTADOS}

Se evaluaron 102 pacientes en total $(n=102)$, los cuales se dividieron en dos grupos para el uso de dispositivos de barrera adicional en la intubación endotraqueal (grupo 1: lámina de plástico, grupo 2: caja de acrílico) (Figura 2) conformado de 51 pacientes cada uno, obteniendo que:

\section{Sociodemográficos:}

1. El promedio de edad para la muestra total fue de $55 \mathrm{DE}$ 15.25 años con una media de $54.47 \mathrm{DE} 16.17$ años para el grupo 1 y $55.54 \mathrm{DE} 14.41$ años en el grupo 2 .

2. En relación al género para el total de la muestra se evidenció que $57.84 \%$ pertenecieron al sexo femenino y $42.16 \%$ al masculino. En el grupo 1 por su parte el $56.86 \%$ correspondió al sexo femenino y 43.14\% al masculino, en el grupo 2 se encontró una frecuencia de $58.82 \%$ y $41.18 \%$ respectivamente.

3. Se observó un promedio de peso de $71.75 \mathrm{DE} 13.30 \mathrm{~kg}$ para el total de la muestra. En el grupo 1 la media de peso fue de $72.54 \mathrm{DE} 13.36 \mathrm{~kg}$ y de $70.96 \mathrm{DE} 13.33 \mathrm{~kg}$ en el grupo 2.

\section{Anestésicos:}

1. Para el Cormack; en la muestra total se obtuvo que en el $70.59 \%$ de los pacientes incluidos en el estudio fueron Cormack I a la videolaringoscopía y no se encontraron pacientes Cormack IV (Figura 3). En el grupo 1 los

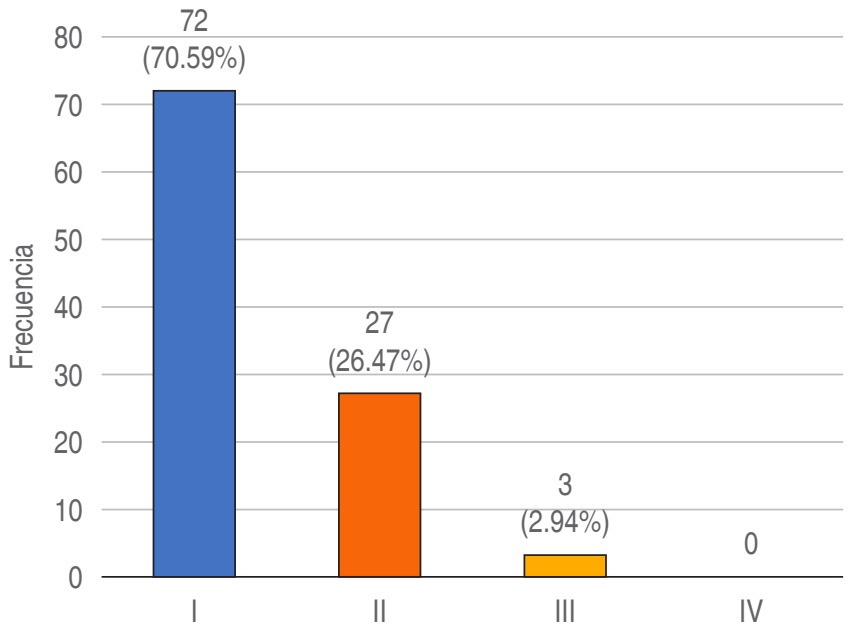

Figura 3: Cormack Lehane.

pacientes con Cormack I correspondieron al 78.43\% (40 pacientes) y 62.75\% (32 pacientes) para el grupo 2.

2. Duración de la laringoscopía: se evidenció en la muestra total una media 62.25 DE 24.06 segundos. Para el grupo 1 la media fue de $54.54 \mathrm{DE} 20.67$ segundos, mientras que en el grupo 2 se encontró 69.96 DE 24.90 segundos.

Se evaluó el tiempo de laringoscopía clasificándolos inicialmente en dos rangos como $\leq 60 \mathrm{y}>$ de 60 segundos donde se observa una diferencia estadística significativa a través de la prueba $\chi^{z}$ de dos colas, IC 95\% (1.63 a 9.53), una p 0.0035 $(<0.05)$ OR 3.94.

Además; se clasificó en tres intervalos la duración de la laringoscopía evidenciando para el total de la muestra en el $57.84 \%$ de los pacientes se logró instrumentar la vía 
aérea en un tiempo menor de 60 segundos (Figura 4). En relación a la duración de la laringoscopía en tres intervalos se encontró que en el grupo 1 el intervalo < 60 segundos fue de $68.63 \%$ ( 35 pacientes) versus $47.06 \%$ ( 24 pacientes) para el grupo 2. En el intervalo de más de 120 segundos no existieron pacientes en el grupo de lámina de plástico; sin embargo, tres pacientes en el grupo de caja de acrílico requirieron este tiempo para asegurar la vía aérea con un tubo endotraqueal, lo que corresponde al $5.88 \%$ del total de pacientes del grupo 2 .

1. Número de intentos de laringoscopía: se obtuvo una media para el total de la muestra de 1.11 DE 0.32 intentos y los hallazgos por grupos mostraron 1.05 DE 0.23 intentos para el grupo 1 versus $1.17 \mathrm{DE} 0.37$ para el grupo 2. En el grupo 1 se aseguró la vía aérea en el $94.12 \%$ (48 pacientes) en el primer intento versus 82.35\% (42 pacientes) en el grupo 2, con un IC de 98.77 y $91.60 \%$ respectivamente. Tres pacientes (5.88\%) del grupo 1 ameritaron un segundo intento de laringoscopía versus nueve pacientes (17.65\%) del grupo 2 (Figura 5). Se evaluó el número de intentos a través de la prueba $\chi^{z}$ de dos colas, IC 95\% una p 0.06 (>0.05) sin significancia estadística, OR 3.42.

2. En la encuesta se evaluaron cinco inconvenientes principalmente observados con el uso de dispositivos de barrera adicional, el hallazgo fue una media de 1.07 (0.91 DE) eventos para el grupo 1 versus $3.27(1.20 \mathrm{DE})$ dificultades para el grupo 2.

La principal dificultad observada en el grupo 1 fue la necesidad de asistencia auxiliar con un $60.78 \%$ (31 casos), el $29.41 \%$ mencionó dificultades para la fijación del tubo endotraqueal y comentaron poca visibilidad en el

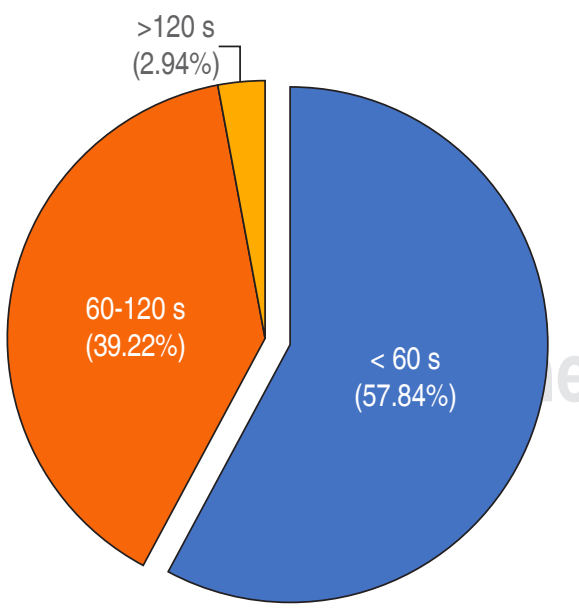

Figura 4: Duración de la laringoscopía.

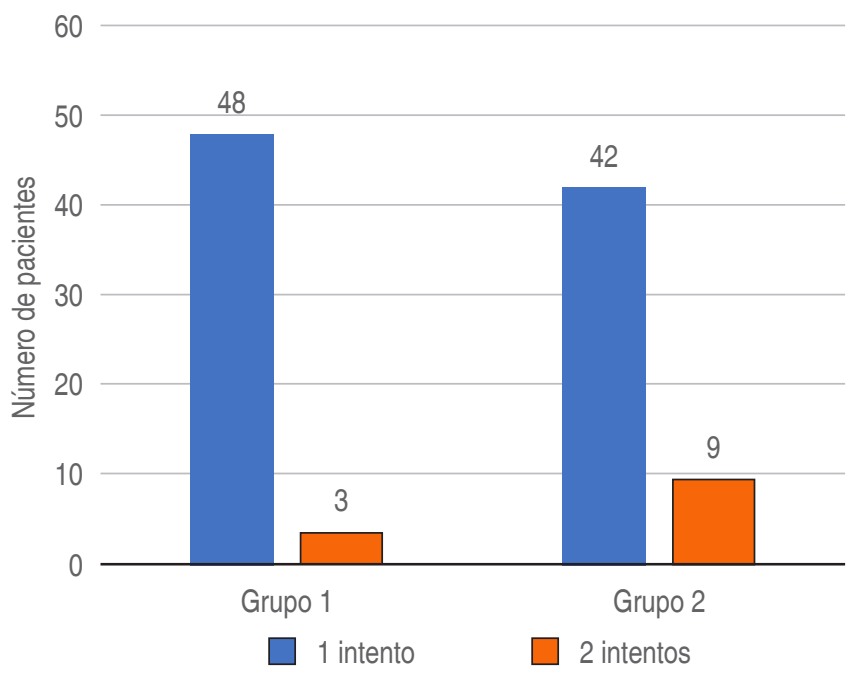

Figura 5: Número de intentos de la laringoscopía.

13.73\% de los casos evaluados. Para este grupo de lámina de plástico no se mencionaron movimientos del dispositivo sobre la mesa ni incomodidad para movilizar los brazos dentro del dispositivo durante la instrumentación de la vía aérea. Solo en dos casos (3.92\%) se manifestó contacto del dispositivo de barrera con el equipo de vía aérea (Tabla 1).

La información en el grupo 2 evidencia que la principal dificultad observada fue también la necesidad de asistencia auxiliar con un $88.24 \%$ (45 casos), se manifestó poca visibilidad en el $52.94 \%$ y la fijación de tubo endotraqueal fue descrita en el 50.98\%. El contacto con el equipo de la vía aérea fue comentado en el 39.22\% y el movimiento sobre la mesa como dificultad estuvo presente en el $37.25 \%$ del total de casos para este grupo de caja de acrílico (Tabla 1).

3. La percepción de satisfacción por el equipo que participó en el manejo de la vía aérea para la muestra total manifestó un 59.80\% (61 pacientes) de las veces sentirse satisfecho con los dispositivos de barrera adicionales para la intubación endotraqueal (Figura 6). El comportamiento por grupos muestra que en el grupo 1, el 27.45\% comentó estar muy satisfecho con la lámina de plástico, el 72.5\% se evaluó satisfecho. Sin embargo en el grupo 2 solo un encuestado manifestó sentirse muy satisfecho (1.96\%) el $47 \%$ satisfecho, $41 \%$ de este grupo estaban pocos satisfechos, insatisfecho el 7.84\% y un participante de la encuesta muy insatisfecho (Tabla 2). Coeficiente de correlación de Pearson para la percepción de dificultad fue de 0.61 expresando una correlación lineal (directa) coeficiente de determinación $37.23 \%$ (> 30 asociación moderada). 


\section{DISCUSIÓN}

Más del 70\% de los pacientes incluidos en este estudio a los que se le realizó intubación orotraqueal con el uso de un dispositivo de barrera adicional se observó Cormack I en la visión videolaringoscópica, no se encontraron pacientes Cormack IV, lo que sugiere no se presentaron dificultades mayores para la instrumentación de la vía aérea. Brown ${ }^{(8)}$ y su equipo, Lai y Chang ${ }^{(9)}$ y Tsai $^{(10)}$ describen distintos dispositivos de barrera flexibles y rígidos para la intubación endotraqueal y aunque estos diseños son innovadores, se asocian a algunos inconvenientes importantes ${ }^{(11)}$. Al igual que en nuestro trabajo, existieron diferencias en el porcentaje de pacientes con Cormack I que correspondieron a $78.4 \%$ para el grupo 1 versus $62.7 \%$ para el grupo 2; sin embargo, tampoco fue posible establecer una asociación directa entre el uso de dispositivo adicional y cambios en la visión laringoscópica.

La duración de la laringoscopía evidenció una diferencia de 15 segundos entre las medianas obtenidas en cada grupo, 54 versus 69 segundos respectivamente, demostrando que el uso de dispositivos de barrera adicional de plástico sobre una mesa de mayo consigue mejores tiempos para el abordaje de la vía aérea, facilitando la manipulación y visualización al realizar procedimientos bajo el dispositivo flexible. Se tienen reportes en otros trabajos de Brown y colegas ${ }^{(8)}$ y Babazade y colaboradores ${ }^{(1)}$ con diseños de cubiertas plásticas tipo cortinas colocadas directamente sobre la cabeza del paciente y manos del operador; sin embargo, el peso de la cubierta plástica suele ser un inconveniente en estos ensayos previos.

Se sustentan las diferencias en el tiempo de laringoscopía al clasificarlas en dos intervalos ( $\leq 60 \mathrm{y}>$ de 60 segundos) donde se obtuvo una diferencia estadística significativa a través de la prueba $\chi^{z}$ una $\mathrm{p}=0.0035$. Cuando se evaluó en tres intervalos, se muestra que para una duración de más de 120 segundos no existieron pacientes en el grupo de lámina de plástico; sin embargo, tres casos en el grupo de la caja de acrílico requirieron este tiempo para asegurar la vía aérea con un tubo endotraqueal, lo que sugiere que los dispositivos rígidos como la caja de acrílico pueden dificultar la manipulación del operador, prolongando los tiempos de instrumentación de la vía aérea.
Con respecto al número de videolaringoscopías por paciente al utilizar dispositivos de barrera adicionales, en su mayoría fueron al primer intento. Los hallazgos por grupos mostraron un menor número de ensayos en el grupo de lámina de plástico con tres versus nueve pacientes del grupo de caja de acrílico que ameritaron un segundo intento de laringoscopía. Lo que podría sugerir mayor dificultad para esta última herramienta; no obstante, no hay significancia estadística para la prueba de $\chi^{z}$ en esta evaluación. Estudios previos están de acuerdo en que uno de los inconvenientes de los diseños rígidos es que obliga al operador a situarse lejos del lado del paciente, lo que limita el manejo de la vía aérea, por lo que aumenta la carga cognitiva y los tiempos de intervención ${ }^{(10)}$.

El número de dificultades encontradas al encuestar al personal que instrumentó la vía aérea se planteó teniendo en cuenta observaciones de estudios anteriores y se mostraron diferencias de 1.07 eventos para el grupo 1 versus 3.27 dificultades para el grupo 2. Para el grupo de lámina de plástico,

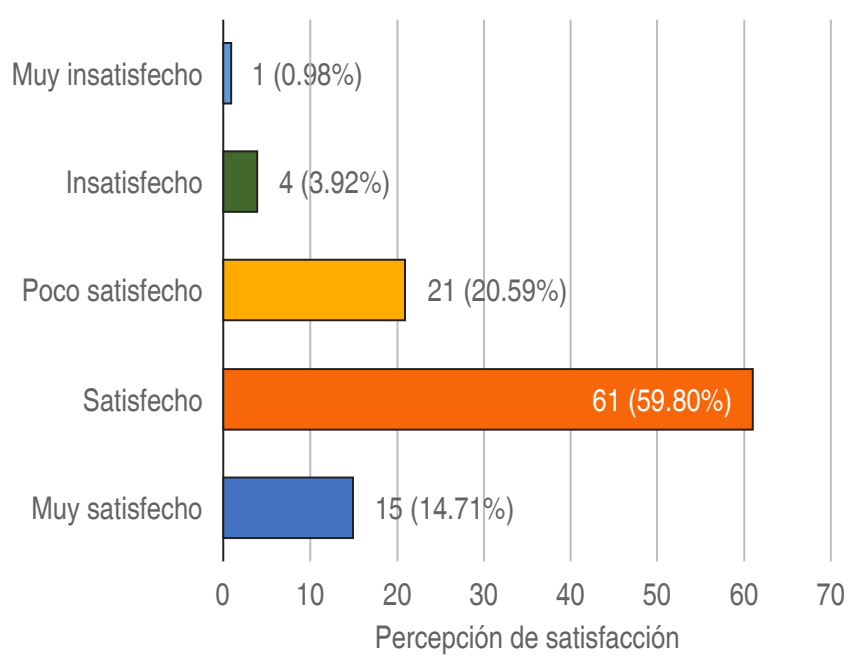

Figura 6: Grado de satisfacción.

La percepción de satisfacción por el equipo que participó en el manejo de la vía aérea para la muestra total manifestó un $59.80 \%$ de las veces sentirse satisfecho con los dispositivos de barrera adicionales para la intubación endotraqueal.

Tabla 1: Dificultades encontradas con el uso de dispositivo de barrera.

\begin{tabular}{lccc} 
Dificultades & $\begin{array}{c}\text { Grupo 1 } \\
\mathrm{n}(\%)\end{array}$ & $\begin{array}{c}\text { Grupo 2 } \\
\mathrm{n}(\%)\end{array}$ & $\begin{array}{c}\text { Total } \\
\mathrm{n}(\%)\end{array}$ \\
\hline Necesidad de asistencia auxiliar & $31(60.78)$ & $45(88.23)$ & $76(74.51)$ \\
Fijación de tubo endotraqueal & $15(29.41)$ & $26(50.9)$ & $41(40.20)$ \\
Incomodidad & $0(0)$ & $30(58.82)$ & $30(29.41)$ \\
Contacto con equipos vía aérea & $2(3.92)$ & $20(39.21)$ & $22(21.50)$ \\
Movimientos sobre la mesa & $0(0)$ & $19(37.25)$ & $19(18.62)$ \\
Ninguna & $14(27.45)$ & $0(0)$ & $14(13.72)$
\end{tabular}




\begin{tabular}{lccr}
\hline \multicolumn{4}{c}{ Tabla 2: comparación grado de satisfacción; } \\
dispositivos flexible vs rígido.
\end{tabular}

los hallazgos más frecuentes fueron la necesidad de asistencia auxiliar, seguida de la dificultad para la fijación del tubo endotraqueal y en menor medida (sólo dos pacientes en este grupo) contacto con equipos de vía aérea, no se mencionaron movimientos del dispositivo sobre la mesa ni incomodidad para movilizar los brazos dentro del mismo. Se realizó prueba $\chi^{z}$ para cada una de estas cinco observaciones con significancia estadística demostrada IC 95\% ( $\mathrm{p}<0.05)$. Lo que sugiere para nuestra evaluación que, al utilizar dispositivos de barrera, de preferencia sean flexibles, para cualquier caso se insiste en que se debe contar con personal auxiliar al momento de la instrumentación de la vía aérea.

En el presente estudio la percepción de satisfacción para el equipo médico marcó una predilección para el uso de dispositivo de barrera tipo lámina de plástico, encontrando que $27.4 \%$ del personal médico manifestó sentirse muy satisfecho y $72.5 \%$ de ellos satisfechos con su utilización. La evaluación de esta herramienta con algunas modificaciones en nuestro ensayo ha sido utilizada previamente, se mantiene las ventajas del diseño de barrera, evidenciando que ofrece un espacio adecuado alrededor de la cabeza y el cuello del paciente, tanto para el laringoscopista como para el asistente de la vía aérea, y que permite proporcionar un manejo efectivo de las vías respiratorias desde la preoxigenación hasta la extubación ${ }^{(11)}$.

\section{CONCLUSIONES}

El personal médico evaluado en este trabajo prefiere la lámina de plástico antes que la caja de acrílico como dispositivo adicional para la intubación orotraqueal. Teniendo en cuenta visión videolaringoscópica, duración y número de intentos de laringoscopía menores, una disminución del número de dificultades para el médico que instrumenta la vía aérea con la lámina plástica, se sugiere utilizar esta estrategia con la ventaja de su cómoda manipulación, económica, reusable y fácilmente disponible.

Consideraciones éticas: El presente protocolo se realizó con base en las Guías ICH (International Conference on Harmonisation of Technical Requirements for Registration of Pharmaceuticals for Human Use) de Buenas Prácticas Clínicas. El consentimiento informado cuenta con los lineamientos especificados en la NOM-SSA-004 del expediente clínico. Asimismo, se adoptaron los lineamientos que establece la NOM-SSA-012, para la ejecución de proyectos de investigación en seres humanos. Se cumple con lo establecido en el reglamento de la Ley General de Salud en Materia de Investigación para la salud de los Estados Unidos Mexicanos en relación a los aspectos éticos en seres humanos, que dispone en el Título Segundo Capítulo Uno del Artículo 13 que toda investigación en la que el ser humano sea sujeto de estudio, deberá prevalecer el criterio del respeto a su dignidad y la protección de sus derechos y bienestar. Además del artículo 14 donde refiere que la investigación que se realice en seres humanos debe desarrollare conforme a las bases allí mencionadas.

El estudio fue validado y autorizado por el Consejo de Ética del Hospital Regional «General Ignacio Zaragoza», siendo asignado el número de registro 293.2020. A su vez cada paciente aceptó y firmó de manera voluntaria el consentimiento informado. Este estudio se encuentra en acuerdo con los principios éticos para la investigación médica en seres humanos, establecidos en la Declaración de Helsinki. Ningún paciente tuvo alguna repercusión al ser partícipe del presente trabajo de investigación.

\section{REFERENCIAS}

1. Babazade R, Khan ES, Ibrahim M, et al. Additional Barrier to Protect Healthcare Workers during Intubation. Anesth Analg. 2020;131:e47-e48.

2. Gorbalenya AE, Baker SC, Baric RS, et al. Severe acute respiratory syndrome-related coronavirus: The species and its viruses a statement of the Coronavirus Study Group. Biorxiv Cold Spring Harb Lab. 2020:1-15.

3. Bampoe S, Odor PM, Lucas DN. Novel coronavirus SARS-CoV-2 and COVID-19. Practice recommendations for obstetric anaesthesia: what we have learned thus far. Int J Obstet Anesth. 2020;43:1-8. Available in: https://doi.org/10.1016/j.ijoa.2020.04.006

4. Go YY, Kim Y, Cheon S, et al. Viral dynamics in mild and severe cases of COVID-19. Elsevier. 2020;20:656-657. Available in: https://doi. org/10.1016/S1473-3099(20)30232-2
5. CDC. Enfermedad por coronavirus 2019 (COVID-19). 2020. Disponible en: https://espanol.cdc.gov/coronavirus/2019-ncov/index.html

6. Canelli R, Connor CW, Gonzalez M, et al. Barrier Enclosure during Endotracheal Intubation. N Engl J Med. 2020;382:1957-1958.

7. Matava CT, Yu J, Denning FS. Clear plastic drapes may be effective at limiting aerosolization and droplet spray during extubation: implications for COVID-19. Can J Anesth. 2020;67:902-904. Available in: https:// doi.org/10.1007/s $12630-020-01649-\mathrm{w}$

8. Brown S, Patrao F, Verma S, et al. Barrier System for Airway Management of COVID-19 Patients. Anesth Analg. 2020;131:e34-e35.

9. Lai YY, Chang CM. A Carton-Made Protective Shield for Suspicious/ Confirmed COVID-19 Intubation and Extubation During Surgery. Anesth Analg. 2020;131:e31-e33. 
10. Tsai PB. Barrier Shields: Not Just for Intubations in Today's COVID-19 World? Anesth Analg. 2020;131:e44-e45.

11. Endersby RVW, Ho ECY, Spencer AO, et al. Barrier Devices for Reducing Aerosol and Droplet Transmission in Coronavirus Disease 2019 Patients: Advantages, Disadvantages, and Alternative Solutions. Anesth Analg. 2020;131:e121-e123.

12. Liu Y-C, Kuo R-L, Shih S-R. COVID-19: The First Documented Coronavirus Pandemic in History. Biomed J. 2020;43: 328-333. Available in: http://www.ncbi.nlm.nih.gov/pubmed/32387617

13. Kang D, Choi H, Kim JH, et al. Spatial epidemic dynamics of the COVID-19 outbreak in China. Int J Infect Dis. 2020;94:96-102. Available in: https://doi.org/10.1016/j.ijid.2020.03.076

14. Johnson NPAS, Mueller J. Updating the accounts: global mortality of the 1918-1920 "Spanish" influenza pandemic. Bull Hist Med. 2002;76:105-115.

15. Kain T, Fowler R. Preparing intensive care for the next pandemic influenza. Crit Care. 2019;23:1-9.

16. Simonsen L, Clarke MJ, Schonberger LB, et al. Pandemic versus Epidemic Influenza Mortality: A Pattern of Changing Age Distribution. J Infect Dis. 1998;178:53-60.

17. Viboud C, Simonsen L, Fuentes R, et al. Global mortality impact of the 1957-1959 influenza pandemic. J Infect Dis. 2015;212:738-745.

18. Li G, Fan Y, Lai Y, et al. Coronavirus infections and immune responses. J Med Virol. 2020;92:424-432. Available in: http://dx.doi.org/10.1002/ jmv. 25685

19. OMS. Situation Report-121. Coronavirus disease COVID-2019. 2020. Available in: https://www.who.int/docs/default-source/coronaviruse/ situation-reports/20200520-covid-19-sitrep-121.pdf?sfvrsn=c4be2ec6_2
20. Li Q, Guan X, Wu P, et al. Early transmission dynamics in Wuhan, China, of novel coronavirus-infected pneumonia. N Engl J Med. 2020;382:1199-1207.

21. Peña-Oteroa D, Díaz-Pérez D, De la Rosa-Carrillo D. Are we ready for the new coronavirus ? Soc Española Neumol y Cirugía Torácica SEPAR. 2020;56:195-196.

22. Lauer SA, Grantz KH, Bi Q, et al. The Incubation Period of Coronavirus Disease 2019 (COVID-19) From Publicly Reported Confirmed Cases: Estimation and Application. Ann Intern Med. 2020;172:577-582.

23. Balibrea JM, Badia JM, Rubio Pérez I, et al. Surgical Management of Patients With COVID-19 Infection. Recommendations of the Spanish Association of Surgeons. Cir Esp (Engl Ed). 2020;98:251-259.

24. Wang D, Hu B, Hu C, et al. Clinical Characteristics of 138 Hospitalized Patients with 2019 Novel Coronavirus-Infected Pneumonia in Wuhan, China. JAMA - J Am Med Assoc. 2020;323:1061-1069.

25. Li W, Huang J, Guo X, et al. Anesthesia Management and Perioperative Infection Control in Patients With the Novel Coronavirus. J Cardiothorac Vasc Anesth. 2021;35:1503-1508.

26. Greenland JR, Michelow MD, Wang L, et al. COVID-19 Infection: Implications for Perioperative and Critical Care Physicians. Anesthesiology. 2020;132:1346-1361. Erratum in: Anesthesiology. 2020;133:693.

27. Ghia S, Lazar M, Epstein J, et al. “Anesthesia Stat” to Intubate a Coronavirus Disease 2019 (COVID-19) Patient: Implications for the Anesthesiologist. J Cardiothorac Vasc Anesth. 2020;34:2566-2570.

28. De la Torre-Anderson J, De la Torre-Buendía J, Zamora-García V. Eficacia y seguridad del sugammadex. Rev Mex Anestesiol. 2014;37:77-82. 